\title{
Color Noise Cancellation by TDBLMS Algorithm Based on Adaptive Wiener Filter
}

\author{
Chanchal Barha ${ }^{1}$, K.S. Solanki ${ }^{2}$ \\ P.G. Scholar Ujjain Eng. College Ujjain (M.P), Affiliated to R.G.P.V. Bhopal (M.P.) ${ }^{1}$ \\ Assistant Professor Ujjain Eng. College Ujjain (M.P), Affiliated to R.G.P.V. Bhopal (M.P.) ${ }^{2}$
}

\begin{abstract}
Image denoising is a kind of processing of image which belongs to image restoration, and the ultimate goal of restoration techniques is to improve an image in some predefined sense. In order to improve the power of signal to noise ratio of image, a local adaptive Wiener filter with Two dimensional block least mean square (TDBLMS) algorithm is applied to a 2-D image. Several 1-D windows could be constructed on the direction character of sub-image in the filter window after wavelet transform. To verify the feasibility of this approach, the simulations in the blockadaptation phase with the block sizes of $4 \times 4,8 \times 8,16 \times 16$, and $32 \times 32$ are performed. The simulation results show that this approach achieves a higher signal-to-noise ratio in each case of block size.
\end{abstract}

Keywords: TBLMS, 2-D, 1-D, PSNR, RMSE, Wiener filter, wavelet transform.

\section{INTRODUCTION}

Image noise: is random variation of brightness or color information in images, and is usually a type of electronic noise. It can be produced by the sensor and circuitry of a scanner or digital camera. Image noise can also originate in film grain and in the unavoidable shot noise of an ideal photon detector. Image noise is an undesirable by-product of image capture that adds spurious and extraneous information.

Main types of noises are:

Gaussian noise: caused by poor illumination and/or high temperature, and/or transmission e.g. electronic circuit noise.

Salt and paper noise: impulsive noise is sometimes called salt-and-pepper noise or spike noise.

Shot noise: typically that caused by statistical quantum fluctuations, that is, variation in the number of photons sensed at a given exposure level. This noise is known as photon shot noise.

Quantization noise: The noise caused by quantizing the pixels of a sensed image to a number of discrete levels is known as quantization noise. Adaptive filters are widely used in various applications for achieving a better performance [1]. In the applications of digital image processing, two dimensional (2-D) adaptive algorithms such as TDLMS, TDBLMS, OBA, OBAI, and TDOBSG are usually used [2][3]. Either in TDLMS or TDBLMS, the convergence factors are constant. Instead of the constant convergence factors in TDLMS and TDBLMS, the space-varying convergence factors are used in OBA, OBAI, and TDOBSG for better convergence performance. However, such space varying convergence factors will increase the computational complexity due to the computations for the new convergence factor of next block.

In this paper, we proposed an adaptive Wiener filter with weight training mechanism by finding a suitable weight (coefficient) matrix for the digital filter in advance. Then, treat this weight matrix as the initial weight matrix for the processing of noise cancellation.

Copyright to IJARCCE

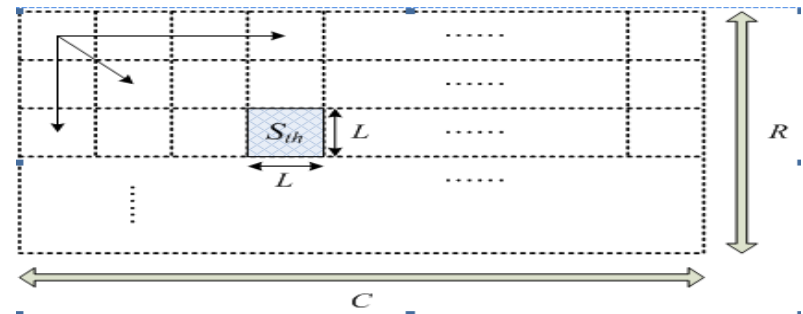

Fig. 1. 2-D block-by-block processing with disjoint square blocks of a dimension $\mathrm{L} x \mathrm{~L}$.

\section{II.TDBLMS ALGORITHM}

An image signal of 2-D is usually partitioned into blocks with a dimension of $L \times L$ for each in the 2-D disjoint block-by-block image processing. An image with $R$ rows of pixel and $C$ columns of pixel partitioned into $\frac{R}{L} \times \frac{C}{L}$ blocks is illustrated in Fig. 1. The relationship between the block index $S$ and the spatial block index $(r, c)$ is [4]

$$
\mathrm{S}=(\mathrm{r}-1) \times(C / L)+\mathrm{c}
$$

Where $r=1, \ldots, R / L$ and $c=1, \ldots, C / L$. For convenient, the $(r, c)$-th element $d(r, c)$ of the image can be treated as the $\left(r_{b}, c_{b}\right)$-th element in the $S$-th block and denoted as the element $d_{s}\left(r_{b}, c_{b}\right)$. The relationship is

$d_{s}\left(r_{b}, c_{b}\right)=\mathrm{d}\left[(\mathrm{r}-1) \mathrm{L}+r_{b},(c-1) L+c_{b}\right]$

Where $r_{b}=1, \ldots, L$ and $c_{b}=1, \ldots, L$

The image is processed block-by-block sequentially from left to right and from top to bottom in which each pixel is convolved the pixel in a filter window with a dimension of $M \times N$. Fig. 2 illustrates this approach which performs the operations from (3) to (5) iteratively [5]. That is,

$y_{s}\left(r_{b}, c_{b}\right)=\sum_{i=1}^{m} \sum_{j=1}^{n} w_{s}(\mathrm{i}, \mathrm{j}) \times X\left[(r-1) L+r_{b}+\right.$

$(M-1)-i,(c-1) L+c_{b}+(\mathrm{N}-1)-\mathrm{j}$ 


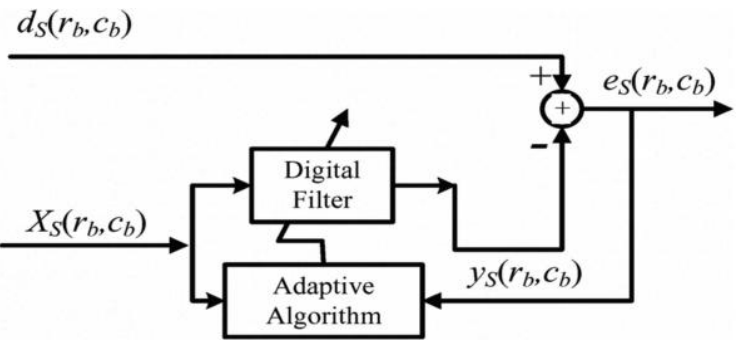

Fig. 2, 2-D adaptive filter.
Where $\mathrm{P}$ is the termination parameter and BNCR stands for the block-noise-cancellation ratio that is defined as

$\mathrm{BNCR}=10 \log \left[\left(\sigma_{X}^{2}-\left(\sigma_{d}^{2}-\sigma_{e}^{2}\right)\right) / \sigma_{X}^{2}\right]$

In (7), $\sigma_{X}^{2}$ stands for the power of the reference signal $X_{S}$ $\left(r_{b}, c_{b}\right)$, and can be expressed as

$\sigma_{X}^{2}=\frac{\sum_{K=1}^{L+M-1} \sum_{L=1}^{L+N-1}\left[X_{S}(K, L)-X_{\text {mean }}\right]}{[L+(M-1)-1][L+(N-1)-1]} ;$

Where $y_{s}\left(r_{b}, c_{b}\right)$ is the image of the $S$-th block after processing, $W(i, j) S$ is the $(i, j)$-th element in the weight matrix $W_{s}$ of the $S$-th block. The error signal $e_{s}\left(r_{b}, c_{b}\right)$ is the difference between the image $y_{s}\left(r_{b}, c_{b}\right)$ and the primary input image $d_{s}\left(r_{b}, c_{b}\right)$. That is,

$$
e_{s}\left(r_{b}, c_{b}\right)=d_{s}\left(r_{b}, c_{b}\right)-y_{s}\left(r_{b}, c_{b}\right)
$$

The updating mechanism of the weight matrix $W_{s+1}$ of the $(S+1)$-th block is expressed as

$$
\begin{aligned}
& w_{s+1(i, j)}=w_{s}(i, j)+\frac{2}{L \times L} \text { 目 } \sum_{r_{b}=1}^{L} \sum_{c_{b}=1}^{L} e_{s}\left(r_{b}, c_{b}\right) \times \\
& X(r b+r L-i, c b+c L-j)
\end{aligned}
$$

Where $\mu$ is the convergence factor.

\section{ADAPTIVE FILTER WITH WEIGHT- TRAINING MECHANISM}

There are two phases in the proposed adaptive filter. They are the weight-training phase and the block-adapting phase. Fig. 3 shows the block diagram of the proposed adaptive filter.

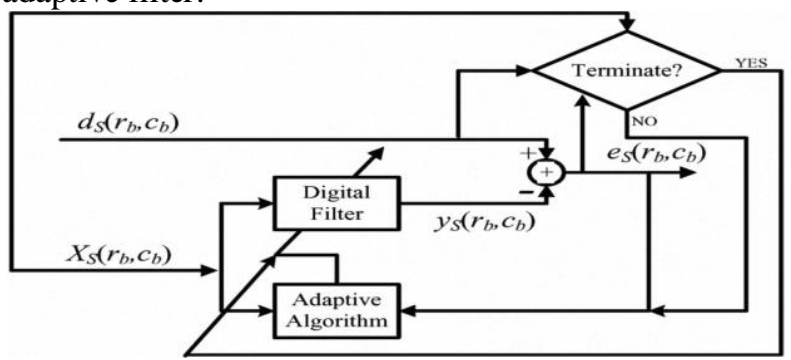

Fig. 3. Adaptive filter with weight-training mechanism.

\section{A. Weight-Training Phase (WTP)}

In order to improve the convergence rate, a suitable weight matrix $W_{T a}$ that will be treated as the initial weight matrix $W_{1}$ for the the processing in the block-adapting phase is found in the weight-training phase. In WTP, all the elements of the initial weight matrix $W_{T 1}$ are set to be zero. That is, $W_{T 1}=\left[W_{T 1}(\mathrm{i}, \mathrm{j})\right] \mathrm{MXN}$ where the element $W_{T 1}(\mathrm{i}, \mathrm{j})=0$ for $\mathrm{i}=1,2, \ldots, \mathrm{M}$ and $\mathrm{j}=1,2, \ldots, \mathrm{N}$. Then, the TDBLMS algorithm is applied to process the original noisy image that will be scanned block-by-block from left to right and from top to down for updating the weight matrix of each block iteratively until the termination criterion is reached [6]. The operations can be expressed in the equations from (3) to (5). Define the termination criterion as

$$
|\mathrm{BNCR}|<\mathrm{P}
$$

the term $\sigma_{d}^{2}$ is the power of the primary input signal $d_{s}$ $\left(r_{b}, c_{b}\right)$, and can be expressed as

$\sigma_{d}^{2}=\frac{1}{(L-1)^{2}} \sum_{r_{b}=1}^{L} \sum_{c_{b}=1}^{L}\left[d_{s}\left(r_{b}, c_{b}\right)-d_{\text {mean }}\right]^{2}$

The term $\sigma_{e}^{2}$; is the power of the error signal $e_{s}\left(r_{b}, c_{b}\right)$, and can be expressed as

$\sigma_{e}^{2}=\frac{1}{(L-1)^{2}} \sum_{r_{b}=1}^{L} \sum_{c_{b}=1}^{L}\left[e_{s}\left(r_{b}, c_{b}\right)-e_{\text {mean }}\right]^{2}$

In $(8)-(10) X_{\text {mean }} d_{\text {mean }}$ and $e_{\text {mean }}$ stand for the means of $X_{s}, d_{s}$ and $e_{s}$ respetively.

\section{B. Block-Adapting Phase (BAP)}

Once the suitable weight matrix $W_{T a}$ in the weight training phase is found, this weight matrix is treated as the initial weight matrix $W_{1}$ in the block-adapting phase (BAP). In this phase, the original noisy image is processed according to the TDBLMS algorithm [7] again for the noise cancellation.

\section{PROPOSED METHODOLOGY}

Taking color image of the subject and performs the following steps for abolition of the noisy signal. In order to improve the power of signal to noise ratio of image, a local adaptive Wiener filter with Two dimensional block least mean square (TDBLMS) algorithm [8] is applied to a 2-D image. In this work we proposed a new two dimensional adaptive filter [9] which will process a color image. Original image will be first convert in to frames of three colors they are red, green, blue of different intensity level then these frames will be process by two dimensional block least mean square filter (TDBLMS) followed by the wiener filter [10] individually as shown in figure.(4) below. Output of three parallel filters will combine at the last to abolish noise from original image, each image is processed in two phases [11]. In the initial weight matrix decision phase, the block-by-block operations with the smaller block size of $4 \times 4$ are applied to the original noisy image for getting the suitable weight matrix that will be used as the initial one for the block-adaptation phase such that a higher signal-to-noise ratio can be achieved [12]. To verify the feasibility of this approach, the simulations in the block-adaptation phase with the block sizes of $4 \times 4,8$ $\times 8,16 \times 16$, and $32 \times 32$ are performed. The simulation results show that this approach achieves a higher signal-tonoise ratio in each case of block size. 


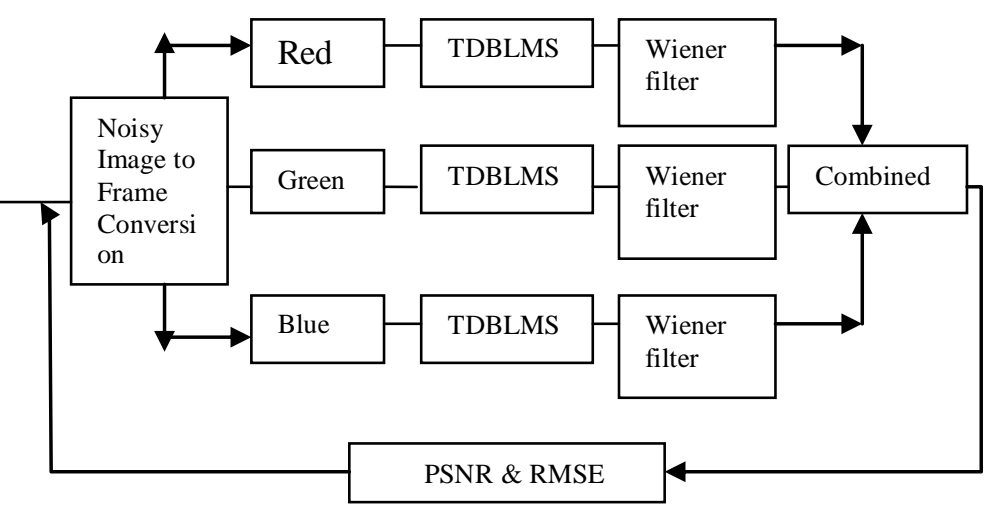

Fig. 4 block diagram of proposed work

\section{SIMULATION RESULTS}

The primary input signal with a dimension of $512 \times$ 512 in the simulation phase is created by adding a Gaussian noise with zero mean and 0.05 variance to the ideal image of church with 512 color-levels in Fig. 5(a) shows the primary input image with a dimension of $512 \mathrm{x}$ 512 and Fig. 5(b) shows the noisy primary input image with an SNR of $0 \mathrm{~dB}$. The convergence factor is $4.5 \mathrm{X} \mathrm{10-}$ 7. For digital filter, the 4-th order transversal FIR filter is chosen to convolved the reference image and the filter window with a dimension of $3 \times 3(\mathrm{M}=3, \mathrm{~N}=3)$.

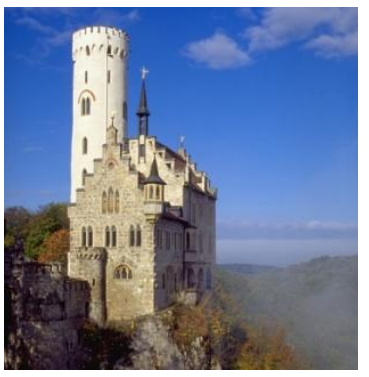

Fig.5 (a)

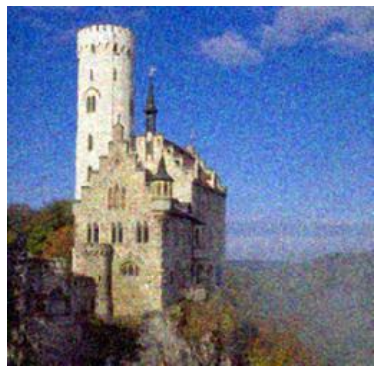

Fig.5 (b)
Fig. 5(a) Primary input image of church with a dimension of 512 X 512 (b) Noisy primary input image with $\mathrm{SNR}=0$ $\mathrm{dB}$. In order to observe the effect of block size on the performance, four different block sizes of $4 \times 4(\mathrm{~L}=4), 8$ x $8(\mathrm{~L}=8), 16 \times 16(\mathrm{~L}=16)$, and $32 \times 32(\mathrm{~L}=32)$ are simulated. Table 1 lists the performance comparison. Fig. 5 (a) is the restored image for the proposed adaptive filter where the termination parameter $\mathrm{P}$ is chosen to be $-10 \mathrm{~dB}$. Fig. 5(b) is the restored image for the proposed adaptive filter where the termination parameter $\mathrm{P}$ is chosen to be $10 \mathrm{~dB}$ The simulation results indicate that the proposed methodology achieves a better performance; however, the performance of the TDBLMS algorithm is not so good for the first several blocks Figure 6 (a), 6(b), 6(c) and 6(d) are showing the simulated results by taking window size or $\mathrm{L}=32,16,8$ and 4 respectively.

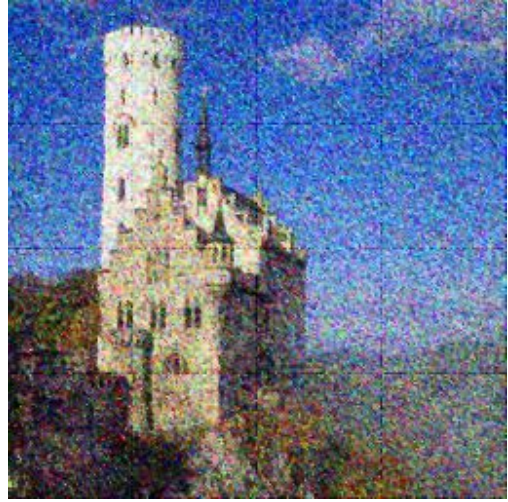

Fig.6 (a) L=32X32

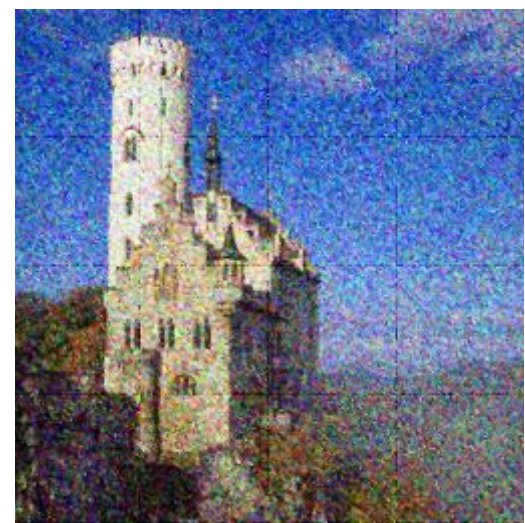

Fig.6 (b) L=16X16

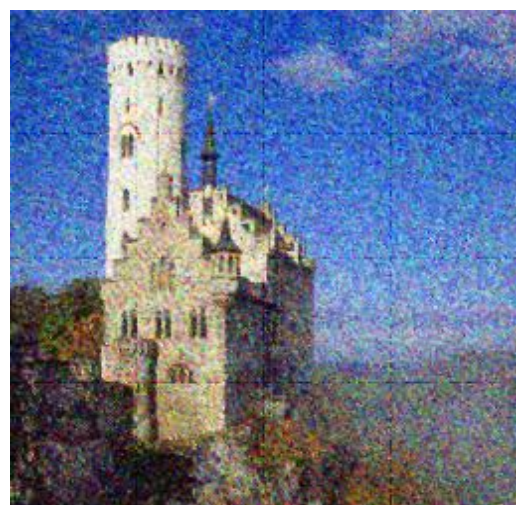

Fig.6 (c) $\mathrm{L}=8 \mathrm{X} 8$

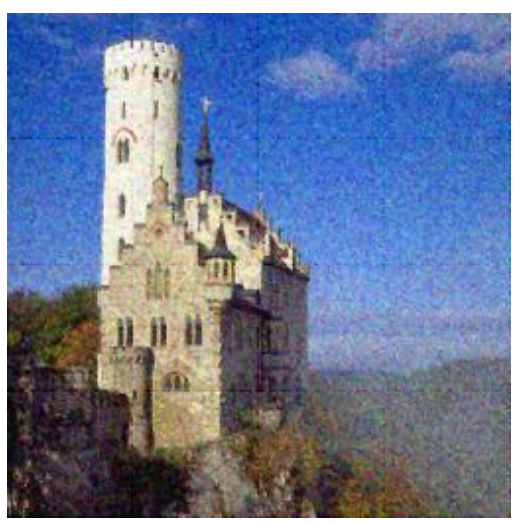

Fig.6 (d) L= 4X4 
Figure 6 Simulation result of predicted image (a) predicted image with $(\mathrm{L}, \mathrm{M}, \mathrm{N})=(32,2,2)$.(b) Predicted image with $(\mathrm{L}, \mathrm{M}, \mathrm{N})=(16,2,2)$. (c) Predicted image with $(\mathrm{L}, \mathrm{M}, \mathrm{N})$ $=(8,2,2)$. (d) Predicted image with $(\mathrm{L}, \mathrm{M}, \mathrm{N})=(4,2,2)$

Table 1 output PSNR and RMSE for adaptive TDLMS noise canceller

\begin{tabular}{|c|c|c|c|c|}
\hline \multirow{3}{*}{$\begin{array}{l}\text { Block } \\
\text { Size } \\
(L \times L)\end{array}$} & \multicolumn{4}{|c|}{ PSNR (db) } \\
\hline & \multicolumn{2}{|c|}{ TDBLMS } & \multicolumn{2}{|c|}{$\begin{array}{c}\text { Proposed } \\
\text { Methodology }\end{array}$} \\
\hline & PSNR & RMSE & PSNR & RMSE \\
\hline $4 \times 4$ & 30.0025 & 22.1645 & 32.3345 & 20.5462 \\
\hline $8 \times 8$ & 28.1452 & 23.3616 & 29.5454 & 22.8586 \\
\hline $16 \times 16$ & 27.6545 & 29.6545 & 29.0011 & 24.4452 \\
\hline $32 \times 32$ & 23.5431 & 35.3514 & 26.8998 & 25.2548 \\
\hline
\end{tabular}

\section{CONCLUSION}

In this work we proposed a new two dimensional adaptive filter which will process a color image. Original image will be first convert in to frames of three colors they are red, green, blue of different intensity level then these frames will be process by two dimensional block least mean square filter (TDBLMS) followed by the wiener filter individually as discussed above in the block diagram shown in figure 4 . In order to observe the effect of block size on the performance, four different block sizes of $4 \times 4$ $(\mathrm{L}=4), 8 \times 8(\mathrm{~L}=8), 16 \times 16(\mathrm{~L}=16)$, and $32 \times 32(\mathrm{~L}=$ 32) are simulated. Shows that this approach can achieve the PSNR's of 32.3345, 29.5454, 29.0011 and 26.8998 for different block sizes of $4 \times 4,8 \times 8,16 \times 16$, and $32 \times 32$. The proposed method provides improved image. The proposed work has been tested on well-known benchmark images, where their PSNR and visual results show the superiority of the proposed technique over the conventional techniques.

\section{REFERENCE}

[1] S. Haykin, Adaptive Filter Theory, 4th ed Englewood Cliffs, NJ: Prentice- Hall, 2001

[2] M. M. Hadhoud and D. W. Thomas, "The two-dimensional adaptive LMS (TDLMS) algorithm", IEEE Trans. Circuits Syst.,vol. 35, pp. 485- 494, May 1988.

[3] T. Wang and C. L. Wang, "A new two-dimensional block adaptive FIR filtering algorithm and its application to image restoration", IEEE Trans. Image Proc., vol. 7, pp. 238-246, Feb, 1998.

[4] T. Wang and C. L. Wang, "A new two-dimensional block adaptive FIR filtering algorithm and its application to image restoration," IEEE Trans. Image Proc, vol. 7, pp. 238-246, 1998.

[5] W. B. Mikhael and S. M. Ghosh, "Two-dimensional block adaptive filtering algorithms," in Proc. of IEEE International Symposium on Circuits and Systems. San Diego, CA, pp. 1219-1222, 1992.

[6] W. B. Mikhael and S. M. Ghosh, "Two-dimensional block adaptive filtering algorithms", in Proc. IEEE Int. Symp. Circuits Syst., San Diego, CA, May 1992, pp. 1219-1222.

[7] M. M. Hadhoud and D. W. Thomas, "The two-dimensional adaptive LMS (TDLMS) algorithm," IEEE Trans. Circuits Syst, vol. 35, pp.485-494, 1988.

[8] C.Yau -Chen and Chin-wen Hsia, "adaptive filter based on TDBLMS algorithm for image noise cancellation". Green Circuits and Systems (ICGCS), 2010 International Conference.

[9] C. S. Burrus, "Block implementation of digital filters", IEEE Trans. Circuits Theory, vol. CT-18, pp. 697-701, Nov, 1971.
[10] S. Haykin, Adaptive Filter Theory, 4th ed Englewood Cliffs, NJ: Prentice-Hall, 2001.

11] W. B. Mikhael and F H. Wu, "A fast block FIR adaptive digital filtering algorithm with individual adaptation of parameters", IEEE Trans. Circuits Syst., vol. 36, pp. 1-10, Jan. 1989.

[12] DONOHO D L. De-Noising by Soft-Threshold .IEEE Transactions on Information Theory, 1995, 41(3):613-627. 